\title{
Copolymerization and thermal study of the new methacrylate derivative of 2,4,6-trichlorophenol
}

\author{
Gökhan Demirci $^{1}$ - Beata Podkościelna ${ }^{1} \cdot$ Andrzej Bartnicki $^{1} \cdot$ Pawel Mergo $^{2}$. \\ Małgorzata Gil $^{2} \cdot$ Onur Çetinkaya ${ }^{2} \cdot$ Barbara Gawdzik $^{1}$
}

Received: 9 March 2016/Accepted: 28 June 2016/Published online: 27 July 2016

(c) The Author(s) 2016. This article is published with open access at Springerlink.com

\begin{abstract}
In this study, 2,4,6-trichlorophenyl methacrylate (TClPhMA) was synthesized by the reaction of methacryloyl chloride with 2,4,6-trichlorophenol in the ice bath condition. The obtained monomer was extracted by chloroform and purified on a chromatography column. In order to confirm the chemical structure of the new compound, spectroscopic studies (ATR-FTIR, ${ }^{1} \mathrm{H}$ NMR, ${ }^{13} \mathrm{C}$ NMR and GC-MS) were undertaken. The obtained TCIPhMA was copolymerized with commercially available monomers such as methyl methacrylate, styrene, 1,4-divinylbenzene and 2-hydroxymethyl methacrylate. The copolymers were obtained by bulk polymerization in which benzoyl peroxide was used as a free radical initiator. The thermal properties of the copolymers were investigated by differential scanning calorimetry and thermogravimetry.
\end{abstract}

Keywords Copolymers - Spectroscopic studies - Thermal properties $\cdot$ Bulk polymerization

\section{Introduction}

Poly(methyl methacrylate) (PMMA) is used in various applications, e.g., immobilization materials, nanocomposites, and sensors [1-4]. PMMA provides excellent

Barbara Gawdzik

barbara.gawdzik@poczta.umcs.lublin.pl

1 Department of Polymer Chemistry, Maria Curie-Sklodowska University, Pl. Marii Curie-Skłodowskiej 5, 20-031 Lublin, Poland

2 Optical Fiber Technology Laboratory, Maria CurieSklodowska University, Pl. Marii Curie-Skłodowskiej 5, 20-031 Lublin, Poland resistance to both chemical and weather corrosion and demonstrates high light transmittance [5]. PMMA is a commercially available polymer which is mostly used in optical fiber technology. Polymer optical fiber (POF) has beneficial properties such as low cost, high ductility, large core diameter, and easy handling characteristics. These infer great potential for their implementation instead of traditional silica glass-based optical fibers for short-length applications. However, a relatively low glass transition temperature ( $\mathrm{Tg}$ ) (around $100{ }^{\circ} \mathrm{C}$ ) and high water absorption are the main disadvantages of PMMA [6-8]. POF applications of PMMA are limited by high losses in the visible and near-infrared region which are dominated by the combinations of stretch and deformation vibrations of $\mathrm{C}-\mathrm{H}$ bonds $[9,10]$. Replacing the hydrogen atoms in $\mathrm{C}-\mathrm{H}$ bond with deuterium and fluorine atoms leads to the minimization of the absorption band because of the decrease in the band vibration energy [11]. According to Imamura et al., the substitution of deuterium for hydrogen improves the transparency at $1.3 \mu \mathrm{m}$ [12]. In the range of telecommunication wavelengths $(1500-1600 \mathrm{~nm})$, the transparency is increased by the replacement of a part of $\mathrm{C}-\mathrm{H}$ bonds by $\mathrm{C}-\mathrm{F}$ bonds [13], also providing refractive index tunability and a decrease in PMMAs sensitivity to moisture [14]. This problem can be solved by already-designed polymers including deuterated fluoromethacrylates or polysiloxanes [12, 15], fluorinated polyimides [14, 16], and perfluorocyclobutane aromatic ether polymers [17]. Unfortunately, these polymers are relatively expensive for both consumer and end user.

In order to increase the optical properties of materials used in optical fiber technology, dopants are applied [18-24]. According to Sengupta et al., the polymers doped with chlorinated dopants showed no absorption peak and retained the broad windows around the source wavelength. 
Fig. 1 Scheme of synthesis of TCIPhMA<smiles>C=C(C)C(=O)Oc1c(Cl)cc(Cl)cc1Cl</smiles>

As reported by Koike et al., DPT (diphenyl-p-tolylamine)doped polymers provided a lower refractive index and higher Tg value. The addition of compounds with chlorine or fluorine atoms influences the transparency and attenuation [21, 24]. For this reason, the creation of new compounds is needed and investigated.

In this study, a new monomer with chlorine atoms in $2,4,6$ substitutes in phenolic derivative is considered. In order to introduce vinyl functionalization, the reaction of phenolic groups with methacrylic acid was carried out. In this way, the new monomer 2,4,6-trichlorophenyl methacrylate (TCIPhMA) was obtained. Next, the copolymerization reactions of TCIPhMA with commercially available monomers such as methyl methacrylate (MMA), styrene (ST), 1,4-divinylbenzene (DVB) and 2-hydroxymethyl methacrylate (HEMA) were investigated.

The effect of different chemical structures of comonomers (aliphatic: MMA and HEMA and aromatic: DVB and ST) on the physico-chemical properties of the obtained copolymers was studied. Thermal properties of the obtained copolymers were investigated using differential scanning calorimetry and thermogravimetry.

Thermal stability of TCIPhMA copolymers have not been published, whereas information on properties of trifunctional methacrylate monomers and their copolymers is available [25]. It can be expected that such polymers will be at least as stable as their analogues without chlorine. As the obtained copolymers may be used in polymer optical fiber technology, the refractive indices for liquid compositions were determined.

\section{Experimental}

\section{Materials}

2,4,6-trichlorophenol, triethyleneamine, methacryloyl chloride, methyl methacrylate, 2-hydroxyethyl methacrylate and dibenzoyl peroxide were purchased from Aldrich.
Styrene, magnesium sulfate, and chloroform were obtained from POCh. 1,4-divinylbenzene was purchased from Merck.

\section{Preparation of trichlorophenyl methacrylate (TCIPhMA)}

In a $500-\mathrm{cm}^{3}$ round bottomed flask equipped with a mechanical stirrer, a thermometer, and a dropper, $0.2 \mathrm{~mol}$ of 2,4,6-trichlorophenol was placed with $35 \mathrm{~mL}$ of triethyleneamine and $0.22 \mathrm{~mol}$ of methacryloyl chloride in the ice bath and stirred for $5 \mathrm{~h}$ (Fig. 1). To remove the water from the product, 0.035 mol magnesium sulfate was added. The obtained trichlorophenyl methacrylate was extracted by chloroform and purified on a chromatography column.

\section{Copolymerization}

Copolymerization of TCIPhMA with MMA, ST, DVB, and HEMA was carried out in glass form (Fig. 2a-d). Composition mass ratio was always $1: 10$, with $1 \%(\mathrm{w} / \mathrm{w})$ benzoyl peroxide (BPO) used as an initiator. The same amount of BPO was applied for all compositions. Copolymers were obtained after 3 steps of polymerization in a water bath and heater chamber. All compositions were kept in a water bath at $70{ }^{\circ} \mathrm{C}$ for $24 \mathrm{~h}$ to initiate polymerization. After this, all copolymers were transferred to a heater chamber and heated at $45^{\circ} \mathrm{C}$ for $24 \mathrm{~h}$. Then, the temperature was increased to $120{ }^{\circ} \mathrm{C}$ and heating continued for $3 \mathrm{~h}$ to crosslink the residual double bound inside the copolymers. All copolymers were kept in the heater chamber until the chamber cooled to room temperature.

\section{Characterization}

ATR-FTIR spectra were recorded on a Brucker TENSOR 27 apparatus (Germany), equipped with diamond crystal. The spectra were gathered from 600 to $4000 \mathrm{~cm}^{-1}$. 
Fig. 2 a Scheme of synthesis of TCIPhMA-MMA, b scheme of synthesis of TCIPhMA-ST, c scheme of synthesis of TCIPhMA-DVB, d scheme of synthesis of TCIPhMA-HEMA<smiles>C=C(C)C(=O)Oc1c(Cl)cc(Cl)cc1Cl</smiles><smiles>COC(=O)C(C)(C)CC(C)(C)C(=O)Oc1c(Cl)cc(Cl)cc1Cl</smiles>

P(TCIPhMA-MMA)

(b)<smiles>C=C(C)C(=O)Oc1c(Cl)cc(Cl)cc1Cl</smiles><smiles>C=Cc1ccccc1</smiles><smiles>O=C(C=CC1CCCC1)C1CCCC1</smiles>

TCIPhMA Styrene (ST)<smiles>CC(C)(C)CC(C)(C)C(=O)Oc1c(Cl)cc(Cl)cc1Cl</smiles>

P(TCIPhMA-ST)

(c)<smiles>C=C(C)C(=O)Oc1c(Cl)cc(Cl)cc1Cl</smiles>

TCIPhMA<smiles>C=Cc1ccc(C=C)cc1</smiles>

DVB<smiles>O=C(CC(=O)c1ccccc1)CC1CCCC1</smiles>

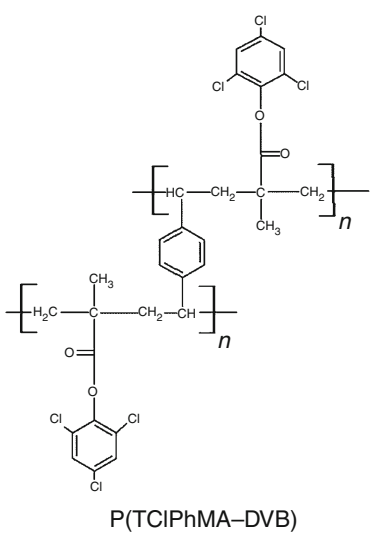

(d)<smiles>C=C(C)C(=O)OCCOC(=O)C(C)(C)CC(C)(C)CC(C)(C)C(=O)Oc1c(Cl)cc(Cl)cc1Cl</smiles>

P(TCIPhMA-HEMA) 
Table 1 Properties of the liquid compositions

\begin{tabular}{lll}
\hline Mixture of monomers (1:10) & Refractive index $N_{\mathrm{D}}^{20}$ & $\begin{array}{l}\text { Density/ } \\
\mathrm{g} \mathrm{cm}^{-3}\end{array}$ \\
\hline Pure MMA & 1.4142 & 0.94 \\
TCIPhMA-MMA & 1.679 & 0.8549 \\
TCIPhMA-ST & 1.676 & 0.8171 \\
TClPhMA-DVB & 1.692 & 0.8289 \\
TClPhMA-HEMA & 1.693 & 0.9824 \\
\hline
\end{tabular}

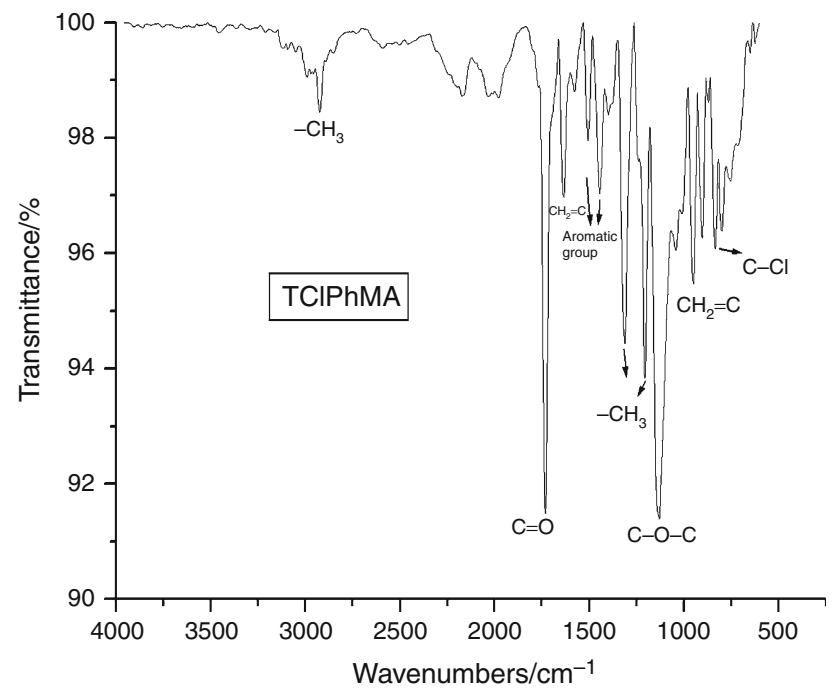

Fig. 3 ATR-FTIR spectra of the new obtained monomer

Proton nuclear magnetic resonance $\left({ }^{1} \mathrm{H}\right.$ NMR) spectra were obtained by Brucker 300 MSL instrument (Brucker, Germany) operating at the ${ }^{1} \mathrm{H}$ resonance frequency of $300 \mathrm{MHz}$. Chemical shifts were performed with deuterated chloroform $\left(\mathrm{CDCl}_{3}\right)$ serving as an internal standard. ${ }^{13} \mathrm{C}$ NMR spectrum of the newly obtained monomer in $\mathrm{CDCl}_{3}$ was obtained using the same apparatus.

Gas chromatography-mass spectroscopy (GC-MS) result was obtained by a Thermo-Finnigan DSQ spectrometer (Finnigan, USA) hyphenated with a gas chromatograph Trace GC-Ultra equipped with a fused-silica Equity-5 capillary column $(20 \mathrm{~m} \times 0.18 \mathrm{~mm}$ I.D., film thickness $0.20 \mu \mathrm{m})$. The conditions were as follows: injector PTV-split 1:20; program temperature $50-320{ }^{\circ} \mathrm{C}$ with the rate $15^{\circ} \mathrm{C} \mathrm{min}{ }^{-1}$; MS electron ionization at $70 \mathrm{eV}$; temperature of ion volume $220{ }^{\circ} \mathrm{C}$.

Differential scanning calorimetry (DSC) curves were obtained with the use of a DSC Netzsch 204 calorimeter (Netzsch, Günzbung, Germany) with the sample mass of $\sim 5-10 \mathrm{mg}$ in close aluminum crucible under nitrogen atmosphere $\left(30 \mathrm{~mL} \mathrm{~min}^{-1}\right)$. Dynamic scans were performed at a heating rate of $10{ }^{\circ} \mathrm{C} \mathrm{min}{ }^{-1}$ in temperature range $20-550{ }^{\circ} \mathrm{C}$.

Thermogravimetry (TG) studies were performed on STA 449 Jupiter F1 instrument, Netzsch (Germany), with the sample mass of $\sim 5-10 \mathrm{mg}$ in open porcelain crucible in both oxidative and inert atmospheres $\left(20 \mathrm{~mL} \mathrm{~min}^{-1}\right)$. In the argon atmosphere, the temperature range was $20-550{ }^{\circ} \mathrm{C}$, whereas in the air the range was $20-600^{\circ} \mathrm{C}$. The loss mass temperatures $\left(T_{5 \%}, T_{20 \%}, T_{50 \%}\right)$, and first, second, and third decomposition temperatures $\left(T_{1}, T_{2}, T_{3}\right)$ were determined.

\section{Results and discussion}

\section{Properties of the liquid compositions}

Properties of the compositions such as refractive index and density are presented in Table 1. All of the liquid compositions have densities close to methyl methacrylate, but their refractive indices are higher than that of commercial methyl methacrylate. This property of the obtained polymers allows them to be used as a core material. Among them, TCIPhMA-ST has the lowest refractive index and density. This result shows that these copolymers can be useful as a core material for POF.

\section{ATR-FTIR spectroscopy}

In order to confirm the chemical formula of the new monomer, ATR-FTIR studies were performed. The ATRFTIR spectrum of the TCIPhMA is presented in Fig. 3. In the spectrum, absorption of $\mathrm{C}-\mathrm{O}-\mathrm{C}$ group gives strong peak at $1128 \mathrm{~cm}^{-1}$. The aromatic skeletal absorption is observed at 1440 and $1633 \mathrm{~cm}^{-1}$. The signal of $\mathrm{C}=\mathrm{O}$ group occurs at $1732 \mathrm{~cm}^{-1}$. The stretching vibrations band of vinyl groups $(\mathrm{C}-\mathrm{H})$ are seen at $942 \mathrm{~cm}^{-1}$ and for methyl groups $(\mathrm{C}-\mathrm{H})$ at $2924 \mathrm{~cm}^{-1}$. A C-H bands are observed at 1232 and $1319 \mathrm{~cm}^{-1}$, whereas the $\mathrm{C}-\mathrm{Cl}$ group occurs at $796 \mathrm{~cm}^{-1}$.

\section{NMR spectroscopy}

In order to identify the structures of monomer, the ${ }^{1} \mathrm{H}$ NMR and ${ }^{13} \mathrm{C}$ NMR spectra were recorded (Fig. $4 \mathrm{a}, \mathrm{b}$ ). In the ${ }^{1} \mathrm{H}$ NMR spectrum of the new obtained monomer, the signals of $-\mathrm{CH}_{2},-\mathrm{CH}_{3}$, and $-\mathrm{CH}$ groups were observed in 5.90 and 6.50, 2.10 and $7.40 \mathrm{ppm}$, respectively. In the ${ }^{13} \mathrm{C}$ NMR spectrum, the most important signal is shown at $165.0 \mathrm{ppm}$. The signal expresses the carbonyl group of ester in the compound. Signals 2 and 3 refer to vinyl group, whereas 5, 6, 7 refer to carbon atoms in aromatic ring. 


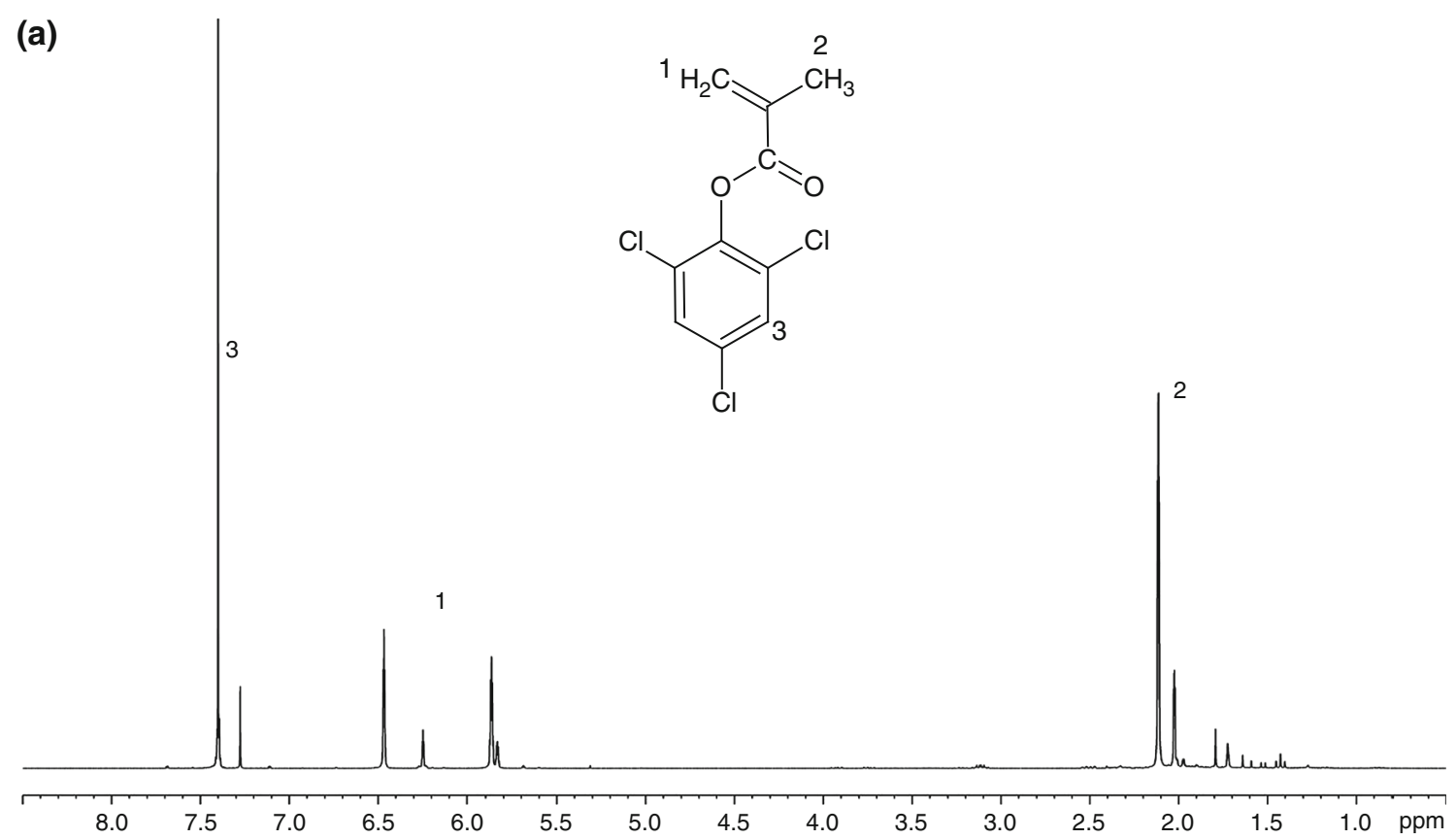

(b)

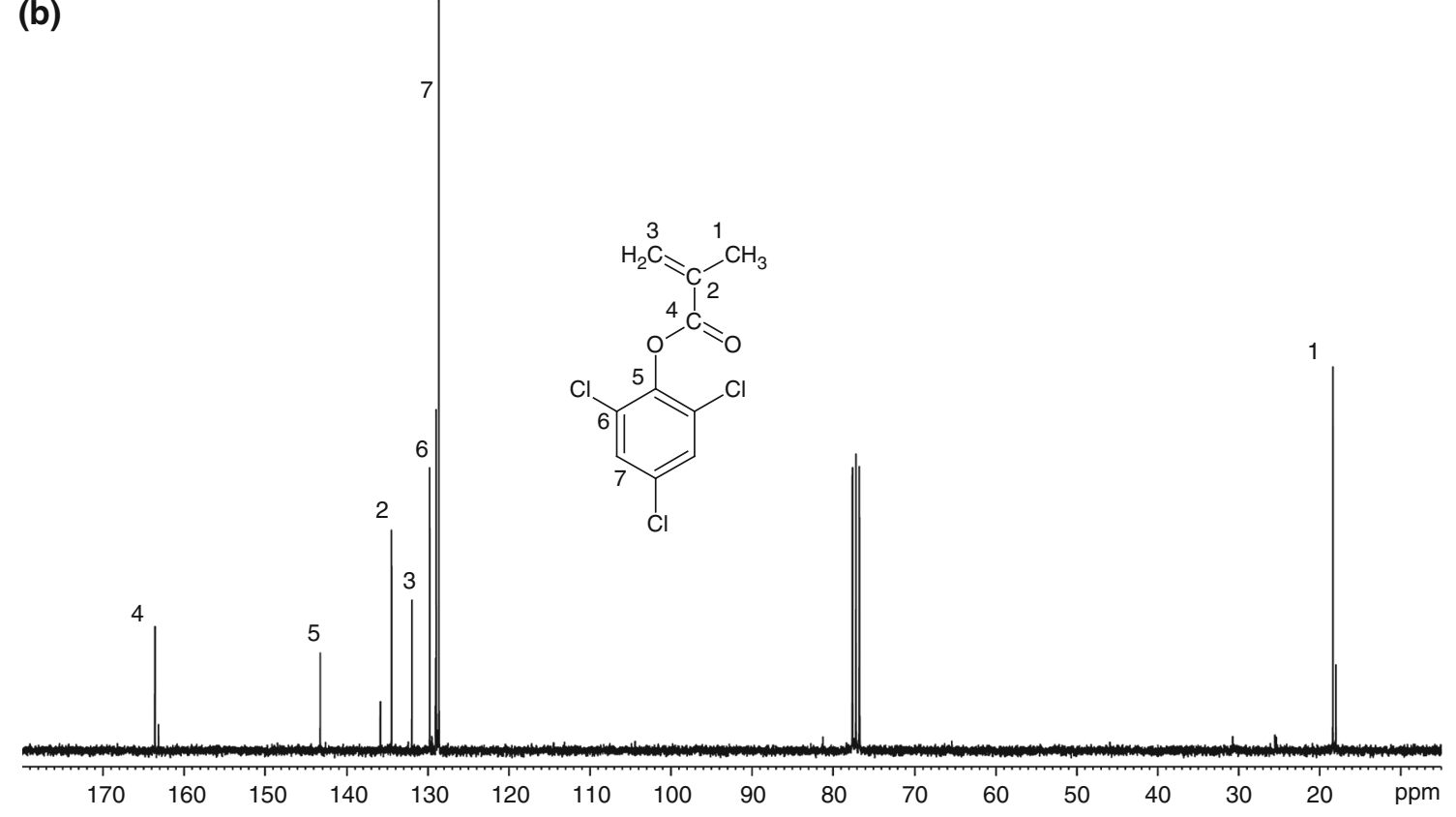

Fig. 4 a ${ }^{1} \mathrm{H}$ NMR spectrum of TClPhMA, $\mathbf{b}{ }^{13} \mathrm{C}$ NMR spectrum of TCIPhMA

\section{GC-MS}

The chemical structure of TClPhMA was additionally confirmed by GC-MS. As its spectrum was not available in the literature, identification was achieved by analyzing molecular and fragmentary ions. In the spectrum presented in Fig. 5, molecular ion corresponding to the calculated molecular weight of TCIPhMA (264) is visible. A large number of fragmentary ions are also noticeable.

\section{Thermogravimetry and DSC analysis}

The DSC curves of the copolymers are presented in Fig. 6 . The DSC analyses show the different thermal behaviors of the studied copolymers. In the range of $100-280{ }^{\circ} \mathrm{C}$ for MMA and HEMA copolymers, some endothermic effects corresponding to the degradation of linear aliphatic fragments are observed [26]. In the case of TCIPhMA-DVB, a small exothermic peak at $160{ }^{\circ} \mathrm{C}$ is visible, probably 


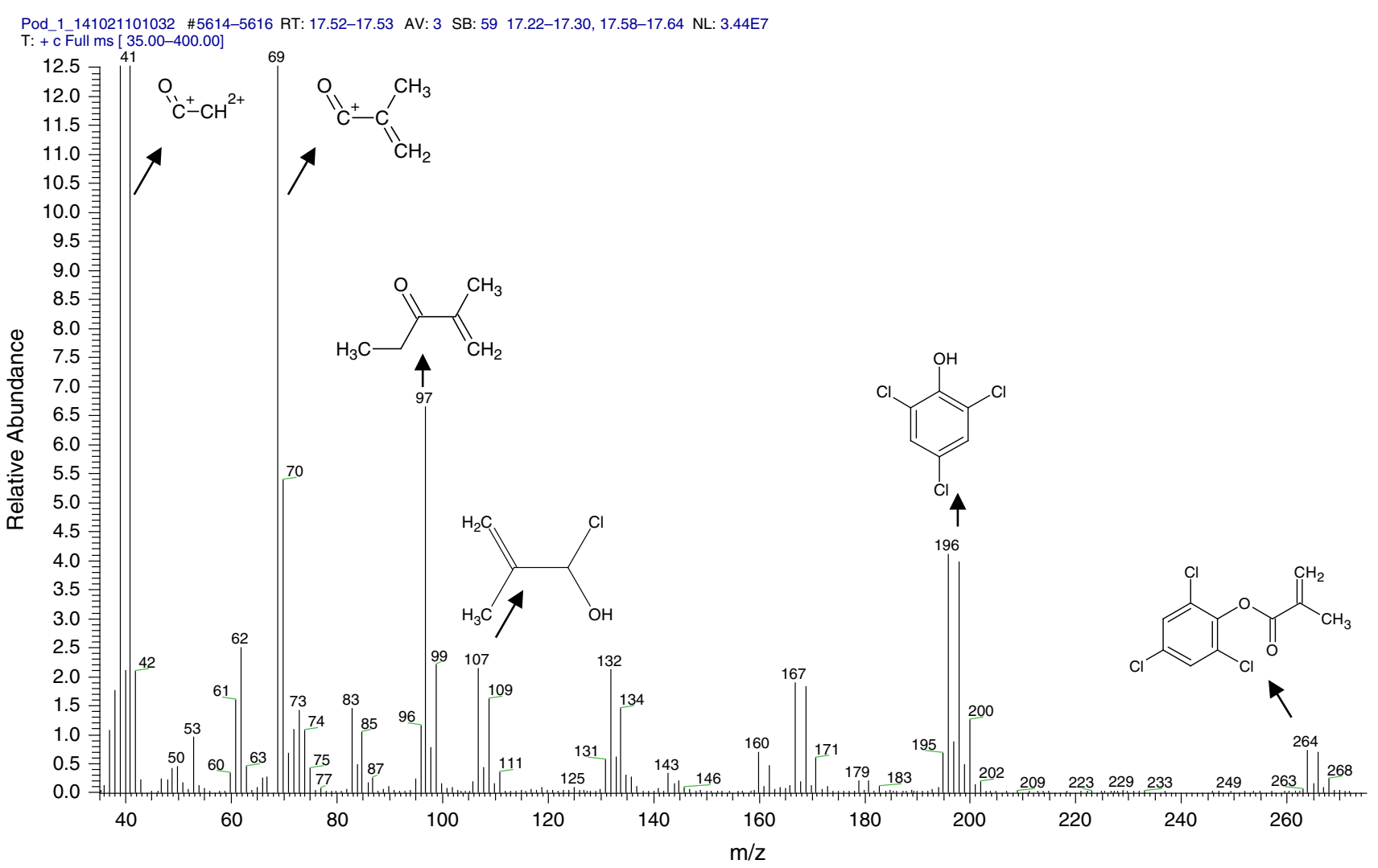

Fig. 5 GC-MS spectrum of the new obtained monomer

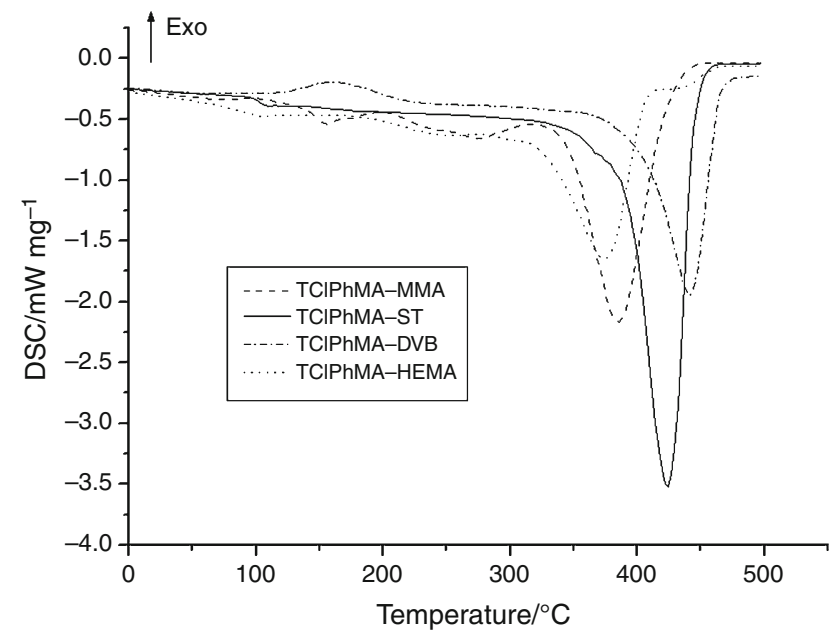

Fig. 6 DSC results of the copolymers

connected with the crosslinking effect of DVB. The endothermic effects related to the thermal degradation of the samples occur at above $360{ }^{\circ} \mathrm{C}$ for TCIPhMA-HEMA, at above $380{ }^{\circ} \mathrm{C}$ for TCIPhMA-MMA, at above $420{ }^{\circ} \mathrm{C}$ TCIPhMA-ST and at above $440{ }^{\circ} \mathrm{C}$ for TClPhMA-DVB polymeric samples. The highest degradation temperature of TCIPhMA-DVB is associated with the aromatic structure

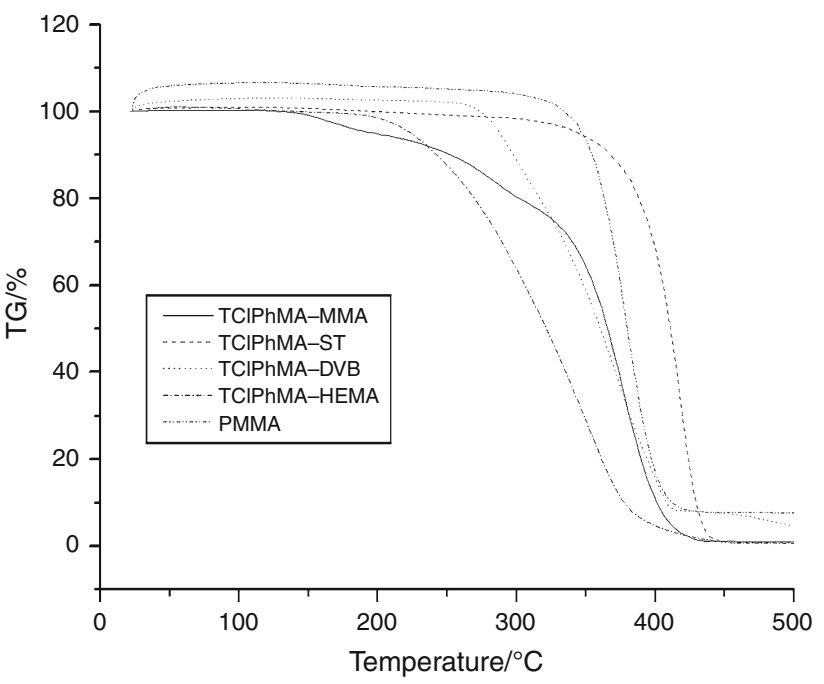

Fig. 7 TG curves of the copolymers in inert condition

and crosslinking character (two vinyl groups) of the DVB comonomer.

Representative results of TG and DTG analyses obtained in an inert condition. TG results are shown in Fig. 7 and collected in Table 2. It can be seen that the $5 \%$ $(\mathrm{w} / \mathrm{w})$ mass loss of copolymer is in the range from 
Table 2 Thermal stabilities of the studied copolymers in inert condition

\begin{tabular}{|c|c|c|c|c|c|c|}
\hline Copolymer (1:10) & $T_{5 \%} /{ }^{\circ} \mathrm{C}$ & $T_{50 \%} /{ }^{\circ} \mathrm{C}$ & $T_{\mathrm{f}} /{ }^{\circ} \mathrm{C}$ & $T_{1} /{ }^{\circ} \mathrm{C}$ & $T_{2} /{ }^{\circ} \mathrm{C}$ & $T_{3} /{ }^{\circ} \mathrm{C}$ \\
\hline TClPhMA-MMA & 197 & 302 & 550 & 164 & 285 & 377 \\
\hline TClPhMA-ST & 345 & 388 & 550 & 367 & 419 & - \\
\hline TCIPhMA-DVB & 291 & 360 & 550 & 109 & 392 & - \\
\hline TClPhMA-HEMA & 224 & 270 & 550 & 238 & 302 & 353 \\
\hline PMMA & 346 & 380 & 426 & 335 & - & - \\
\hline
\end{tabular}

$T_{5 \%}, T_{50 \%}$ the mass loss rates, $T_{1}, T_{2}, T_{3}$ the decomposition temperatures, $T_{\mathrm{f}}$ the final decomposition temperature
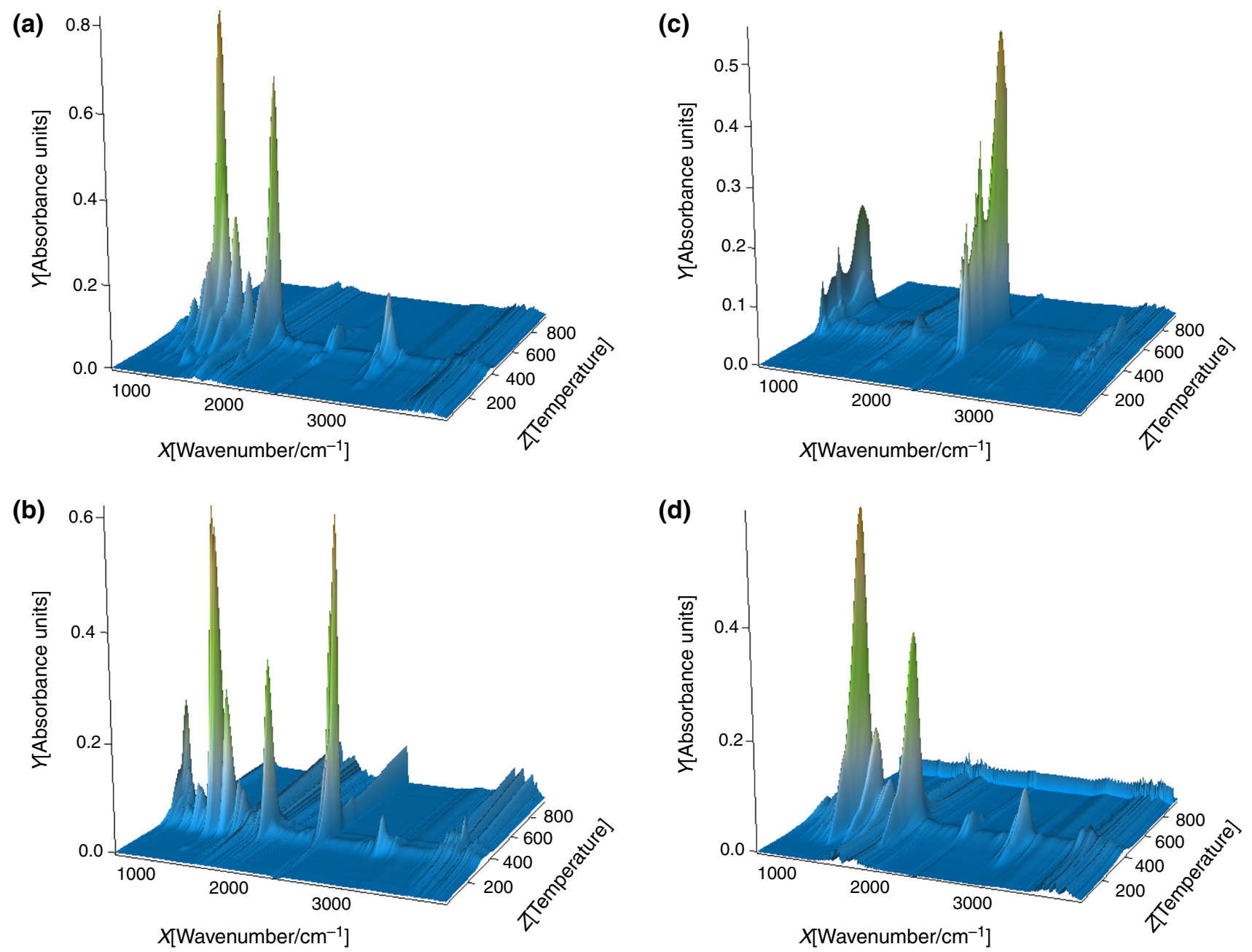

Fig. 8 TG-FTIR graphics of TCIPhMA-MMA (a), TCIPhMA-ST (b), TCIPhMA-DVB (c), TCIPhMA-HEMA (d)

196.6 ${ }^{\circ} \mathrm{C}$ for TClPhMA-MMA, to $345.1^{\circ} \mathrm{C}$ for TClPhMAST, $291.1^{\circ} \mathrm{C}$ for TClPhMA-DVB, $223.9^{\circ} \mathrm{C}$ for TCIPhMA-HEMA and $346{ }^{\circ} \mathrm{C}$ for commercial PMMA. The highest thermal resistance exhibits the TCIPhMA-ST copolymer.

TG-FTIR measurements were taken in an inert condition and presented in Fig. 8a-d. In the case of TCIPhMA-MMA, evaporation of unreacted MMA is observed at $178^{\circ} \mathrm{C}$, whereas its depolymerization occurred at $290-390{ }^{\circ} \mathrm{C}$. Depolymerization of TCIPhMAST occurs at $346{ }^{\circ} \mathrm{C}$, and degradation of the copolymer starts at $400{ }^{\circ} \mathrm{C}$. In the case of TCIPhMA-DVB, evaporation of unreacted TClPhMA occurs at $178{ }^{\circ} \mathrm{C}$. Decomposition of this copolymer-crosslinked network starts at $320{ }^{\circ} \mathrm{C}$ and goes through 3 maxima (357, 457, $605{ }^{\circ} \mathrm{C}$ ). As main volatile products, $\mathrm{CO}_{2}, \mathrm{CO}$ and $\mathrm{H}_{2} \mathrm{O}$ 
Table 3 Thermal stabilities of the studied copolymers in oxidative condition

\begin{tabular}{llllll}
\hline Copolymer $(1: 10)$ & $T_{5 \%} /{ }^{\circ} \mathrm{C}$ & $T_{50 \%} /{ }^{\circ} \mathrm{C}$ & $T_{\mathrm{f}} /{ }^{\circ} \mathrm{C}$ & $T_{1} /{ }^{\circ} \mathrm{C}$ & $T_{2} /{ }^{\circ} \mathrm{C}$ \\
\hline TCIPhMA-MMA & 284 & 341 & 401 & 263 & - \\
TCIPhMA-ST & 300 & 432 & 550 & 273 & - \\
TCIPhMA-DVB & 288 & 360 & 510 & 276 & - \\
TClPhMA-HEMA & 222 & 295 & 393 & 189 & 332 \\
PMMA & 287 & 320 & 398 & 276 & - \\
\hline
\end{tabular}

$T_{5 \%}, T_{50 \%}$ the mass loss rates, $T_{1}, T_{2}, T_{3}$ the decomposition temperatures, $T_{\mathrm{f}}$ the final decomposition temperature

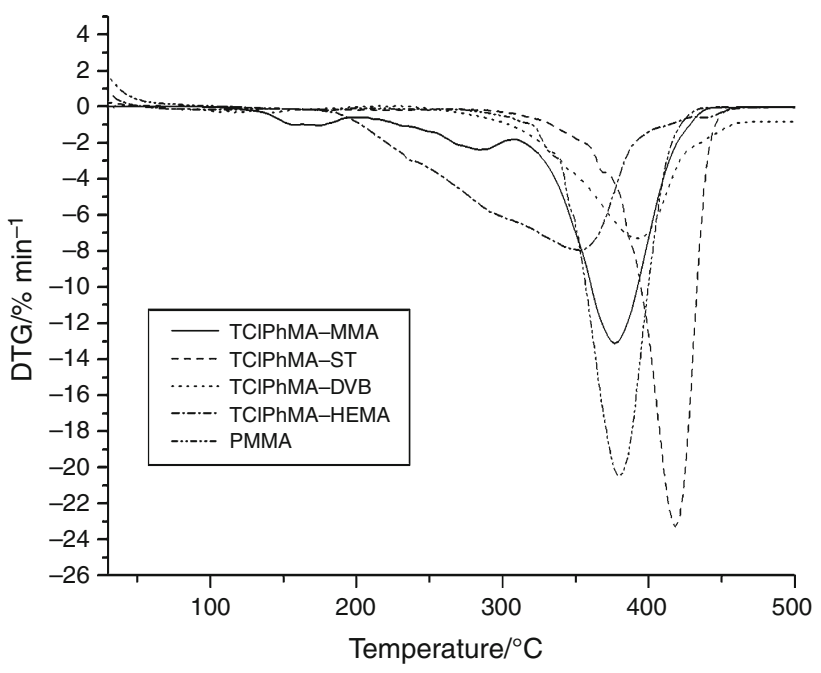

Fig. 9 DTG curves of the copolymers in inert condition

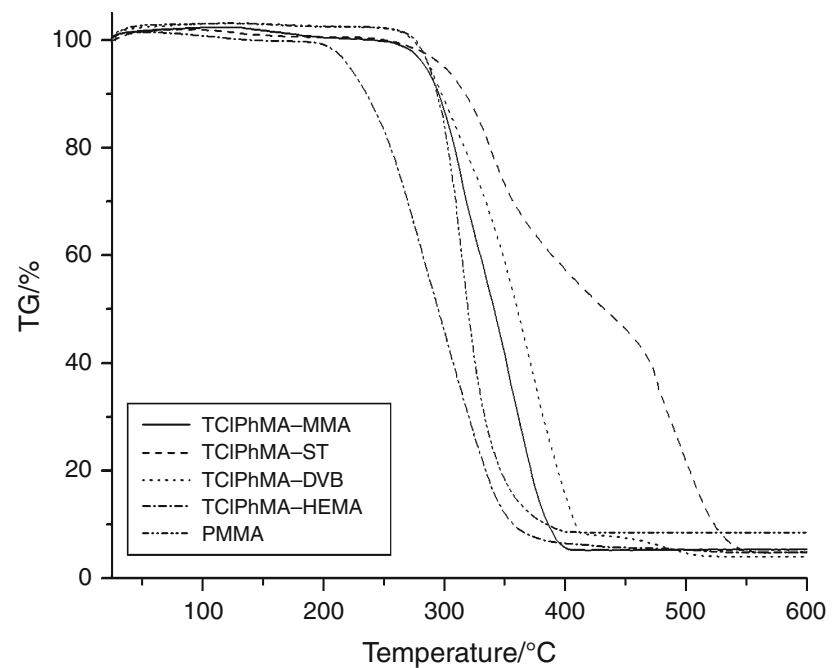

Fig. 10 TG curves of the copolymers in oxidative condition

are observed. For TCIPhMA-HEMA, evaporation of moisture takes place at $100{ }^{\circ} \mathrm{C}$. Its depolymerization is observed at $230-380{ }^{\circ} \mathrm{C}$.

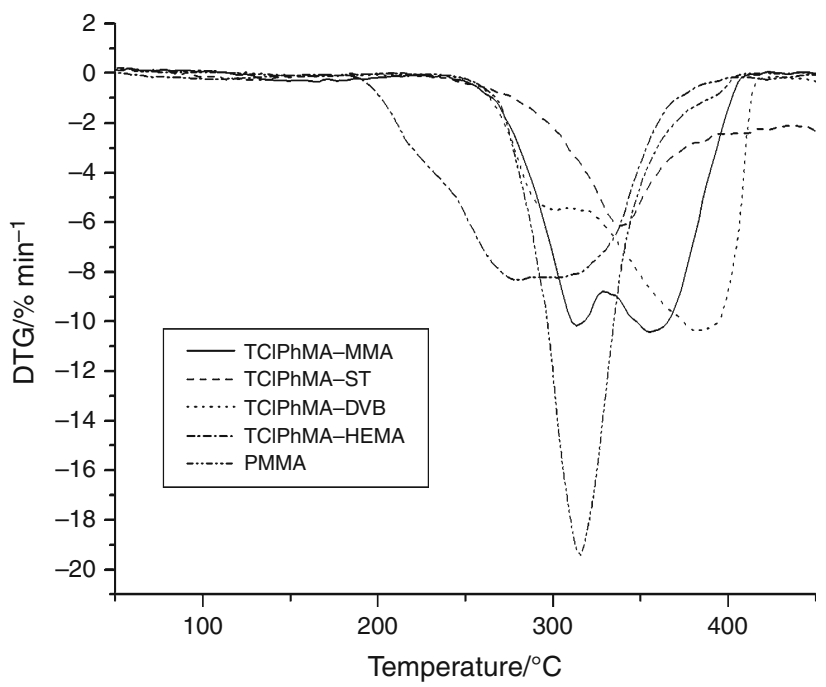

Fig. 11 DTG curves of the copolymers in oxidative condition

The results of DTG measurements are presented in Fig. 9. In the case of TCIPhMA-MMA copolymer, the DTG curve contains three separated degradation steps. The first, second, and third decomposition peaks are observed in the range $150-190,260-290$, and $380-390{ }^{\circ} \mathrm{C}$, respectively. A decomposition peak for TCIPhMA-ST is observed at $420^{\circ} \mathrm{C}$, whereas for TCIPhMA-DVB in the range $390-410{ }^{\circ} \mathrm{C}$. For TCIPhMA-HEMA, the decomposition peak is observed in the range of $350-360{ }^{\circ} \mathrm{C}$ with the maximum mass loss. The obtained TG/DTG results confirm the results obtained earlier by DSC analysis.

For overall study of copolymer thermal properties, TGDTG analyses in oxidative conditions were also carried out. The obtained results are presented in Figs. 10 and 11, as well as the datas are collected in Table 3. It can be seen that the starting mass loss of copolymer ranges from $210{ }^{\circ} \mathrm{C}$ for the TCIPhMA-HEMA, $280{ }^{\circ} \mathrm{C}$ for the TCIPhMA-DVB, TCIPhMA-MMA and PMMA, to $340{ }^{\circ} \mathrm{C}$ for the TCIPhMA-ST. The final decomposition temperature is in the range $360-550{ }^{\circ} \mathrm{C}$. 
Comparing the course of the curves obtained in the inert and oxidative conditions, one can see that up to $180^{\circ} \mathrm{C}$ the studied copolymers are stable. As expected, their thermal resistances in an oxidative condition are lower. The most rapidly decomposed copolymer (TCIPhMA-HEMA) possesses linear aliphatic fragments in its structure.

\section{Conclusions}

Synthesis of the TCIPhMA, its copolymerization with commercial compounds such as MMA, ST, DVB and HEMA of different chemical structures and their physical and thermal properties are presented. TCIPhMA-ST and TCIPhMA-DVB copolymers due to their low refractive indices have potential application in polymer optical fiber technology. However, DVB as tetrafunctional monomer leads to crosslinked polymer and therefore TCIPhMADVB cannot be used in POF technology.

Considering the conditions of POF drawing process, TClPhMA-ST copolymer which additionally indicates the highest thermal resistance is the most suitable for drawing fibers. The obtained 2,4,6-trichlorophenyl methacrylate can be used as a dopant compound that reduces fibers attenuation.

Acknowledgements The research leading to these results has received funding from the People Programme (Marie Curie Actions) of the European Union's Seventh Framework Programme FP7/20072013/under REA Grant Agreement No 608382. The authors wish to thank MSc. Christian Broadway for his English correction of this paper, Carlos III University of Madrid, Madrid, Spain.

Open Access This article is distributed under the terms of the Creative Commons Attribution 4.0 International License (http://crea tivecommons.org/licenses/by/4.0/), which permits unrestricted use, distribution, and reproduction in any medium, provided you give appropriate credit to the original author(s) and the source, provide a link to the Creative Commons license, and indicate if changes were made.

\section{References}

1. Zaharieva J, Milanova M, Todorovsky D. Poly(methylmethacrylate) as immobilization matrix for europium $\beta$-diketonates-morphology and fluorescent properties. Appl Surf Sci. 2011;257:6858-66.

2. Arica MY, Yavuz H, Patir S, Denizli A. Immobilization of glucoamylase onto spacer-arm attached magnetic poly(methylmethacrylate) microspheres: characterization and application to a continuous flow reactor. J Mol Catal B Enzym. 2000;11:127-38.

3. Lewis S, Haynes V, Wheeler-Jones R, Sly J, Perks RM, Piccirillo L. Surface characterization of poly(methylmethacrylate) based nanocomposite thin films containing $\mathrm{Al}_{2} \mathrm{O}_{3}$ and $\mathrm{TiO}_{2}$ nanoparticles. Thin Solid Films. 2010;518:2683-7.

4. Yoo HY, Bruckenstein S. A novel quartz crystal microbalance gas sensor based on porous film coatings. A high sensitivity porous poly(methyl methacrylate) water vapor sensor. Anal Chim Acta. 2013;785:98-103.
5. Yuichi K, Kazuo K, Koichi N. Synthesis of N-cyclohexylmaleimide for heat-resistant transparent methacrylic resin. J Appl Polym Sci. 1997;63:363-8.

6. Baumer S, editor. Handbook of plastic optics. Eindhoven: Wiley; 2005.

7. Zubia J, Arrue J. Plastic optical fibers: an introduction to their technological processes and applications. J Opt Fiber Technol. 2001;7:101-40.

8. Weinert A, editor. plastic optical fibres: principles, components, installations. Erlangen: Publicis MCD; 1999.

9. Groh W. Overtone absorption in macromolecules for polymer optical fibers. Makromol Chem. 1988;189:2861-70.

10. Groh W, Zimmermann A. What is the lowest refractive index of an organic polymer? Macromolecules. 1991;24:6660-3.

11. Lou L, Koike Y, Okamoto Y. Synthesis and properties of copolymers of methyl methacrylate with 2,3,4,5,6-pentafluoro and 4-trifluoromethyl 2,3,5,6-tetrafluoro styrenes: an intrachain interaction between methyl ester and fluoro aromatic moieties. J Polym Sci Part A Polym Chem. 2010;48:4938-42.

12. Imamura $S$, Yoshimura $R$, Izawa $T$. Polymer channel waveguides with low loss at $1.3 \mu \mathrm{m}$. Electron Lett. 1991;27:1342.

13. Boutevin B, Rousseau A, Bosc D. New halogenated monomers and polymers for low loss plastic optical fiber. Fiber Integr Opt. 1994;13(3):309.

14. Matsuura T, Ando S, Sasaki S, Yamamoto F. Low loss, heatresistant optical waveguides using new fluorinated polyimides. Electron Lett. 1993;29(3):269-71.

15. Usui M, Hikita M, Watanabe T, Amano M, Sugarawa S, Hayashida S, Imamura S. Low-loss passive polymer optical waveguides with high environmental stability. J Lightwave Technol. 1996;14(10):2338.

16. Beuhler A, Wargowski DA, Singer KD, Kowalczyk T. Fabrication of low loss polyimide optical waveguides using thin-film multichip module process technology. IEEE T Compon Pack B. 1995;18(2):232.

17. Fischbeck G, Moosburger R, Kostrzewa C, Achen A, Peterman K. Singlemode optical waveguides using a high temperature stable polymer with low losses in the $1.55 \mu \mathrm{m}$ range. Electron Lett. 1997;33(6):518-9.

18. Sharafudeen KN, Adithya A, Vijayakumar S, Sudheesh P, Kalluraya $\mathrm{B}$, Chandrasekharan $\mathrm{K}$. Multiphoton absorption process and self-focusing effect in coumarin derivative doped PMMA films by z-scan and optical limiting studies. Curr Appl Phys. 2011;11:1089-93.

19. Peng GD, Xiong Z, Chu PL. Photosensitivity and gratings in dyedoped polymer optical fibers. Opt Fiber Technol. 1999;5:242-51.

20. Luo Y, Wua W, Wanga T, Cheng X, Zhang Q, Peng GD, Zhu B. Analysis of multimode BDK doped POF gratings for temperature sensing. Opt Commun. 2012;285:4353-8.

21. Koike K, Suzuki A, Makino K, Koike Y. Effects of para-fluorine substituent of polystyrene on gradient-index fiber-optic properties. Opt Mater. 2015;39:143-7.

22. Tama HY, Pun C-FJ, Zhou G, Cheng X, Tse MLV. Special structured polymer fibers for sensing applications. Opt Fiber Technol. 2010;16:357-66.

23. Yu J, Tao X, Tam H. Fabrication of UV sensitive single-mode polymeric optical fiber. Opt Mater. 2006;28:181-8.

24. Sengupta A, Koike K, Koike Y, Okamoto Y. New insights into dopant design for graded-index plastic optical fibers for transmission at $850 \mathrm{~nm}$. Opt Mater. 2014;36:782-6.

25. Kim JG, Chung CM. Trifunctional methacrylate monomers and their photocured composites with reduced curing shrinkage, water sorption, and water solubility. Biomaterials. 2003;24:3845-51.

26. Podkościelna B. Synthesis, spectroscopic and thermal characterization of the new photoluminescent monomer. J Therm Anal Calorim. 2016;123:273-82. 